\title{
The boundedness of multilinear operators on generalized Morrey spaces over the quasi-metric space of non-homogeneous type
}

\section{Xiangxing $\mathrm{TaO}^{1 *}$ and Sha $\mathrm{He}^{2}$}

${ }^{*}$ Correspondence:

xxtao@zust.edu.cn

${ }^{1}$ Department of Mathematics,

Zhejiang University of Science and

Technology, Hangzhou, Zhejiang

310023, P.R. China

Full list of author information is

available at the end of the article

\begin{abstract}
In this paper we study the multilinear fractional integral operators, the multilinear Calderón-Zygmund operators and the multi-sublinear maximal operators defined on the quasi-metric space with non-doubling measure. We obtain the boundedness of these operators on the generalized Morrey spaces over the quasi-metric space of non-homogeneous type.

MSC: 42B20; 42B25; 42B35
\end{abstract}

Keywords: multilinear Calderón-Zygmund operator; multilinear fractional integral; multi-sublinear maximal function; generalized Morrey spaces; non-doubling measure

\section{Introduction and main results}

The boundedness of fractional integral operators on the classical Morrey spaces was studied by Adams [1], Chiarenza and Frasca et al. [2]. In [2], by establishing a pointwise estimate of fractional integrals in terms of the Hardy-Littlewood maximal function, they showed the boundedness of fractional integral operators on the Morrey spaces. In 2005, Sawano and Tanaka [3] gave a natural definition of Morrey spaces for Radon measures which might be non-doubling but satisfied the growth condition, and they investigated the boundedness in these spaces of some classical operators in harmonic analysis. Later on, Sawano [4] defined the generalized Morrey spaces on $\mathbb{R}^{n}$ for non-doubling measure and showed the properties of maximal operators, fractional integral operators and singular operators in this space.

Simultaneously, in 1999, Kenig and Stein [5] gave the boundedness for multilinear fractional integrals on Lebesgue spaces. In 2002, Grafakos and Torres [6] obtained the boundedness for multilinear Calderón-Zygmund operators on Lebesgue spaces. From then on, the theory on multilinear integral operators has attracted much attention as a rapidly developing field in harmonic analysis. Recently, the authors have studied the boundedness of multilinear fractional integrals on Herz-Morrey spaces in [7-10] and the boundedness of multilinear Calderón-Zygmund operators on the Morrey-type spaces in [11-14]. Particularly, the authors $[7,11,13,15]$ established the boundedness for the multilinear operators on Morrey spaces over $\mathbb{R}^{n}$ with non-doubling measures. In this paper, we focus on the

(C) 2013 Tao and He; licensee Springer. This is an Open Access article distributed under the terms of the Creative Commons Attribution License (http://creativecommons.org/licenses/by/2.0), which permits unrestricted use, distribution, and reproduction in any medium, provided the original work is properly cited. 
multilinear operators on generalized Morrey spaces over quasi-metric space $(X, \rho, \mu)$ of non-homogeneous type and extend the works in $[3-7,11,16]$.

Let $(X, \rho)$ be a quasi-metric space with the quasi-metric function $\rho: X \rightarrow[0, \infty)$ satisfying the conditions:

(1) $\rho(x, y)>0$ for all $x \neq y$, and $\rho(x, x)=0$ for all $x \in X$.

(2) There exists a constant $a_{0} \geq 1$ such that $\rho(x, y) \leq a_{0} \rho(y, x)$ for all $x, y \in X$.

(3) There exists a constant $a_{1} \geq 1$ such that

$$
\rho(x, y) \leq a_{1}(\rho(x, z)+\rho(z, y))
$$

for all $x, y, z \in X$.

Here we point out that there is no notion of dyadic cubes on the quasi-metric space and so the method for $\mathbb{R}^{n}$ used in [15] does not work on $(X, \rho)$. Recently, Hytönen [17] introduced the notion of geometrically doubling space.

Definition 1.1 The quasi-metric space $(X, \rho)$ is called geometrically doubling if there exists some $N_{0} \in \mathbb{N}$ such that any ball $B(x, r) \subset X$, where $B(x, r):=\{y \in X: \rho(x, y)<r\}$ with the center $x$ and the radius $r$, can be covered by at most $N_{0}$ balls $B\left(x_{i}, r / 2\right)$ with $x_{i} \in B(x, r)$.

Remark 1.2 Similarly as Hytönen showed in Lemma 2.3 in [17], one can deduce that if the quasi-metric space $(X, \rho)$ is geometrically doubling, then, for any $\delta \in(0,1)$, any ball $B(x, r) \subset X$ can be covered by at most $N_{0} \delta^{-n}$ balls $B\left(x_{i}, \delta r\right)$ with $x_{i} \in B(x, r)$, where $n=$ $\log _{2} N_{0}$.

Given a Borel measure $\mu$ on the quasi-metric space $(X, \rho)$ such that $\mu$ is finite on bounded sets, and let $(X, \rho)$ be geometrically doubling, then continuous, boundedly supported functions are dense in $L^{p}(X, \mu)$ for $p \in[1, \infty)$. See Proposition 3.4 in [17] for details.

The above triple $(X, \rho, \mu)$ will be called a quasi-metric space of non-homogeneous type if the measure $\mu$ satisfies the following growth condition,

$$
\mu(B(x, r)) \leq C_{0} r
$$

with the constant $C_{0}$ independent of the ball $B(x, r) \subset X$. The set of all balls $B \subseteq X$ satisfying $\mu(B)>0$ is denoted by $\mathscr{B}(\mu)$. We know that the analysis on non-homogeneous spaces plays important roles in solving the Painlevé problem as well as the Vitushkin conjecture $[18,19]$. For motives of developing analysis on non-homogeneous spaces and more examples, one could see [20].

Now we give the definition of the generalized Morrey space over $(X, \rho, \mu)$, which is a generalization of the classical Morrey space. Here we remark that Morrey spaces play important roles in the study of partial differential equations.

Definition 1.3 Let $1 \leq p<\infty$ and a function $\phi:(0, \infty) \rightarrow(0, \infty)$ be such that $r^{\frac{1}{p}} \phi(r)$ is non-decreasing. The generalized Morrey space $L^{p, \phi}(X, k, \mu)$ over $X$, where $k>a_{1}$, is defined as

$$
L^{p, \phi}(X, k, \mu):=\left\{f \in L_{\mathrm{loc}}^{p}(\mu):\|f\|_{L^{p, \phi}(X, k, \mu)}<\infty\right\}
$$


with the norm $\|f\|_{L^{p, \phi}(X, k, \mu)}$ given by

$$
\|f\|_{L^{p, \phi}(X, k, \mu)}:=\sup _{B \in \mathscr{B}(\mu)} \frac{1}{\phi(\mu(k B))}\left(\frac{1}{\mu(k B)} \int_{B}|f(x)|^{p} d \mu(x)\right)^{\frac{1}{p}}
$$

where $k B$ is the ball with the same center and $k$ times radius of the ball $B$.

In case $\phi(r)=r^{-\frac{1}{q}}, 1 \leq p<q<\infty$, the space $L^{p, \phi}(X, k, \mu)$ becomes the classical Morrey space $L^{p, q}(X, k, \mu)$ over $X$. Particularly, $L^{p, p}(X, k, \mu)=L^{p}(X, \mu)$.

Remark 1.4 It is worth to point out that if $k_{1}, k_{2}>a_{1}$, then $L^{p, \phi}\left(X, k_{1}, \mu\right)$ and $L^{p, \phi}\left(X, k_{2}, \mu\right)$ coincide as a set and their norms are mutually equivalent. This can be observed by the same arguments used in [4]. For the sake of convenience, we provide the detail. Let $a_{1}<$ $k_{1} \leq k_{2}$. Then the inclusion $L^{p, \phi}\left(X, k_{1}, \mu\right) \subseteq L^{p, \phi}\left(X, k_{2}, \mu\right)$ is obvious by that fact that $r^{\frac{1}{p}} \phi(r)$ is non-decreasing. To see the reverse inclusion, let $f \in L^{p, \phi}\left(X, k_{2}, \mu\right)$ and $B(x, r) \in \mathscr{B}(\mu)$ be fixed. It is sufficient to estimate

$$
I=\frac{1}{\phi\left(\mu\left(B\left(x, k_{1} r\right)\right)\right)}\left(\frac{1}{\mu\left(B\left(x, k_{1} r\right)\right)} \int_{B(x, r)}|f(x)|^{p} d \mu(x)\right)^{\frac{1}{p}} .
$$

The geometrically doubling condition shows that the ball $B(x, r)$ can be covered by at most $N=N_{0} \delta^{-n}$ balls $B\left(x_{i}, \delta r\right)$ with $x_{i} \in B(x, r)$ for any $\delta \in(0,1)$. Moreover, by the quasi-triangle inequality (1.1), we can see that $B\left(x_{i}, k_{2} \delta r\right) \subseteq B\left(x, k_{1} r\right)$ if we choose $0<\delta<\left(k_{1}-a_{1}\right) /\left(a_{1} k_{2}\right)$. Thus,

$$
\begin{aligned}
I^{p} & \leq \sum_{i=1}^{N} \frac{1}{\phi\left(\mu\left(B\left(x, k_{1} r\right)\right)\right)^{p} \mu\left(B\left(x, k_{1} r\right)\right)} \int_{B\left(x_{i}, \delta r\right)}|f(x)|^{p} d \mu(x) \\
& \leq \sum_{i: B\left(x_{i}, \delta r\right) \in \mathscr{B}(\mu)} \frac{1}{\phi\left(\mu\left(B\left(x_{i}, k_{2} \delta r\right)\right)\right)^{p} \mu\left(B\left(x_{i}, k_{2} \delta r\right)\right)} \int_{B\left(x_{i}, \delta r\right)}|f(x)|^{p} d \mu(x) \\
& \leq N\left(\|f\|_{L^{p, \phi}\left(X, k_{2}, \mu\right)}\right)^{p},
\end{aligned}
$$

which implies that $L^{p, \phi}\left(X, k_{1}, \mu\right)=L^{p, \phi}\left(X, k_{2}, \mu\right)$ for any $k_{1}, k_{2}>a_{1}$. With this fact in mind, we sometimes omit parameter $k$ in $L^{p, \phi}(X, k, \mu)$, i.e., write it by $L^{p, \phi}(X, \mu)$.

In this article, we consider the multilinear fractional integral operator, the multilinear Calderón-Zygmund operator and the multi-sublinear maximal operator. The multilinear fractional integral is defined by

$$
\mathcal{I}_{\alpha, m}\left(f_{1}, \ldots, f_{m}\right)(x)=\int_{X^{m}} \frac{f_{1}\left(y_{1}\right) \cdots f_{m}\left(y_{m}\right)}{\left(\rho\left(x, y_{1}\right)+\cdots+\rho\left(x, y_{m}\right)\right)^{m-\alpha}} d \mu\left(y_{1}\right) \cdots d \mu\left(y_{m}\right)
$$

where $0<\alpha<m$. When $m=1$, we denote $\mathcal{I}_{\alpha, m}$ by $\mathcal{I}_{\alpha}$.

Let $\mathcal{T}$ be a multilinear operator initially defined on the $m$-fold product of Schwartz spaces and taking values into the space of tempered distributions. Following [6], we say that $\mathcal{T}$ is an $m$-linear Calderón-Zygmund operator if it extends to a bounded multilinear operator from $L^{p_{1}}(X, \mu) \times L^{p_{2}}(X, \mu) \times \cdots \times L^{p_{m}}(X, \mu)$ to $L^{p}(X, \mu)$ for some $1 \leq p_{1}, \ldots, p_{m}<$ 
$\infty$ and $\frac{1}{p}=\frac{1}{p_{1}}+\frac{1}{p_{2}}+\cdots+\frac{1}{p_{m}}$, and if there exists a kernel function $\mathcal{K}$, the so-called multilinear Calderón-Zygmund kernel, defined away from the diagonal $x=y_{1}=\cdots=y_{m}$ in $X^{m+1}$, satisfying

$$
\mathcal{T}\left(f_{1}, \ldots, f_{m}\right)(x)=\int_{X^{m}} \mathcal{K}\left(x, y_{1}, \ldots, y_{m}\right) f_{1}\left(y_{1}\right) \cdots f_{m}\left(y_{m}\right) d \mu\left(y_{1}\right) \cdots d \mu\left(y_{m}\right)
$$

for all $x \notin \bigcap_{i=1}^{m} \operatorname{supp} f_{i}$, where $f_{i}$ 's are smooth functions with compact support; and the kernel function $\mathcal{K}$ satisfies the size condition

$$
\left|\mathcal{K}\left(x, y_{1}, y_{2}, \ldots, y_{m}\right)\right| \leq C\left(\sum_{i=1}^{m} \rho\left(x, y_{i}\right)\right)^{-m}
$$

and some smoothness conditions; see $[6,16]$ for details. In fact, as for the $m$-linear Calderón-Zygmund operator $\mathcal{T}$, we assume that, by a similar argument as that in $[6,16]$ for the case $X=\mathbb{R}^{n}$, if $\frac{1}{p}=\frac{1}{p_{1}}+\frac{1}{p_{2}}+\cdots+\frac{1}{p_{m}}$, then the $m$-linear Calderón-Zygmund operator $\mathcal{T}$ satisfies

$$
\mathcal{T}: L^{p_{1}}(X, \mu) \times L^{p_{2}}(X, \mu) \times \cdots \times L^{p_{m}}(X, \mu) \rightarrow L^{p}(X, \mu)
$$

for any $1<p_{1}, p_{2}, \ldots, p_{m}<\infty$.

We will also consider the multi-sublinear maximal operator $\mathcal{M}_{\kappa}$, for $\kappa>a_{1}^{2}$, defined by

$$
\mathcal{M}_{\kappa}\left(f_{1}, \ldots, f_{m}\right)(x)=\sup _{x \in B \in \mathscr{B}(\mu)} \prod_{i=1}^{m} \frac{1}{\mu(\kappa B)} \int_{B}\left|f_{i}\left(y_{i}\right)\right| d \mu\left(y_{i}\right) .
$$

In case $m=1$, we denote it by $M_{\kappa}$.

The main result of this paper can be stated as follows.

Theorem 1.5 Let $0<\alpha<m$ and $1<p_{i}<\infty$, and let $\frac{1}{q}=\frac{1}{p_{1}}+\cdots+\frac{1}{p_{m}}-\alpha>0$. For each $i=1, \ldots, m$, let $\phi_{i}:(0, \infty) \rightarrow(0, \infty)$ satisfy

$$
\frac{1}{C_{1}} \leq \frac{\phi_{i}(t)}{\phi_{i}(r)} \leq C_{1} \quad \text { if } 1 \leq \frac{t}{r} \leq 2
$$

and

$$
\int_{r}^{\infty} t^{\alpha / m-1} \phi_{i}(t) d t \leq C_{2} r^{\alpha / m} \phi_{i}(r)
$$

with positive constants $C_{1}$ and $C_{2}$ independent of $r>0$. Then there exists a constant $C$ independent of any admissible $f_{i}$ such that

$$
\left\|\mathcal{I}_{\alpha, m}\left(f_{1}, \ldots, f_{m}\right)\right\|_{L^{q, \psi}(X, \mu)} \leq C \prod_{i=1}^{m}\left\|f_{i}\right\|_{L^{p_{i}}, \phi_{i}(X, \mu)},
$$

where $\psi(t)=t^{\alpha} \phi_{1}(t) \phi_{2}(t) \cdots \phi_{m}(t)$. 
If we take $\phi_{i}(t)=t^{-\frac{1}{l_{i}}}$ and $0<l_{i}<\frac{m}{\alpha}$, then $\phi_{i}$ satisfies conditions (1.4) and (1.5). We remark that if condition (1.4) is replaced by

$$
\phi_{i}(u) \leq C_{3} \phi_{i}(v) \quad \text { for } u \geq v
$$

with the constant $C_{3}>0$, then the theorem is also valid. This can be seen from the proof of the theorem in the next section. Theorem 1.5 yields the following corollary.

Corollary 1.6 Let $0<\alpha<m$ and $1<p_{i}<\infty$, and let $\frac{1}{q}=\frac{1}{p_{1}}+\cdots+\frac{1}{p_{m}}-\alpha>0$. Let $0<l_{i}<\frac{m}{\alpha}$ and $\frac{1}{h}=\frac{1}{l_{1}}+\cdots+\frac{1}{l_{m}}-\alpha$. Then there exists a constant $C$ independent of $f_{i}$ such that

$$
\left\|\mathcal{I}_{\alpha, m}\left(f_{1}, \ldots, f_{m}\right)\right\|_{L^{q, h}(X, \mu)} \leq C \prod_{i=1}^{m}\left\|f_{i}\right\|_{L^{p_{i},}, l_{i}(X, \mu)}
$$

Theorem 1.7 Let $1<p_{i}<\infty$ and $\frac{1}{p}=\frac{1}{p_{1}}+\cdots+\frac{1}{p_{m}}<1$. If the functions $\phi_{i}:(0, \infty) \rightarrow(0, \infty)$ satisfy condition (1.4) or (1.6), and satisfy

$$
\int_{r}^{\infty} \phi_{i}(t)^{\frac{p_{i}}{p}} \frac{d t}{t} \leq C_{4} \phi_{i}(r)^{\frac{p_{i}}{p}}
$$

with the constant $C_{4}$ independent of $r>0$, then there exists a constant $C$ independent of any admissible $f_{i}$ such that

$$
\left\|\mathcal{T}\left(f_{1}, \ldots, f_{m}\right)\right\|_{L^{p, \phi}(X, \mu)} \leq C \prod_{i=1}^{m}\left\|f_{i}\right\|_{L^{p_{i}}, \phi_{i}(X, \mu)}
$$

where $\phi(t)=\phi_{1}(t) \phi_{2}(t) \cdots \phi_{m}(t)$.

Here we point out that if each $f_{i} \in L^{p_{i}}(X, \mu) \cap L^{p_{i}, \phi_{i}}(X, \mu)$, then the multilinear CalderónZygmund operator $\mathcal{T}\left(f_{1}, \ldots, f_{m}\right)$ is well defined, and we will prove estimate (1.8) with the absolute constant $C$ independent of these admissible functions. More remarks on the admissibility for $f_{i} \in L^{p_{i}, \phi_{i}}(X, \mu)$ will be given in Remark 3.1 in Section 3.

Observe that $\phi_{i}(t)=t^{-\frac{1}{l_{i}}}$, for any $0<l_{i}<\infty$, satisfies the conditions in the theorem, thus the corollary follows.

Corollary 1.8 Let $1<p_{i}<\infty$ and $\frac{1}{p}=\frac{1}{p_{1}}+\cdots+\frac{1}{p_{m}}<1$. Let $0<l_{i}<\infty$ and $\frac{1}{l}=\frac{1}{l_{1}}+\cdots+\frac{1}{l_{m}}$. Then there exists a constant $C$ independent of $f_{i}$ such that

$$
\left\|\mathcal{T}\left(f_{1}, \ldots, f_{m}\right)\right\|_{L^{p, l}(X, \mu)} \leq C \prod_{i=1}^{m}\left\|f_{i}\right\|_{L^{p_{i}, l_{i}(X, \mu)}} .
$$

Theorem 1.9 Assume that $\mathcal{M}_{\kappa}$ is a multi-sublinear maximal operator. Let $1<p_{i}<\infty, \frac{1}{p}=$ $\frac{1}{p_{1}}+\cdots+\frac{1}{p_{m}}<1$, and $\phi_{i}$ satisfy condition (1.6). Then there exists a constant $C$ independent of any admissible $f_{i}$ such that

$$
\left\|\mathcal{M}_{\kappa}\left(f_{1}, \ldots, f_{m}\right)\right\|_{L^{p, \phi}(X, \mu)} \leq C \prod_{i=1}^{m}\left\|f_{i}\right\|_{L^{p_{i}, \phi_{i}(X, \mu)}},
$$

where $\phi(t)=\phi_{1}(t) \cdots \phi_{m}(t)$. 
We notice that the results above are new even for the case of Euclidean spaces. Throughout this paper, the letter $C$ always denotes a positive constant that may vary at each occurrence but is independent of the essential variable.

\section{Proof of Theorem 1.5}

Let us first give some requisite theorems and lemmas.

Theorem 2.1 [21] Let $0<\alpha<1,1<p<\frac{1}{\alpha}$ and $\frac{1}{q}=\frac{1}{p}-\alpha$, then the operator $\mathcal{I}_{\alpha}$ is bounded from $L^{p}(X, \mu)$ into $L^{q}(X, \mu)$ if and only if $\mu(B(x, r)) \leq C r$, where the constant $C$ is independent of $x$ and $r$.

Lemma 2.2 [13] Suppose that $\mu$ is a Borel measure on $X$ with the growth condition (1.2). Let $\frac{1}{q}=\frac{1}{p_{1}}+\cdots+\frac{1}{p_{m}}-\alpha>0$ with $0<\alpha<m$ and $1 \leq p_{j} \leq \infty$. Then

(a) if each $p_{j}>1$,

$$
\left\|\mathcal{I}_{\alpha, m}\left(f_{1}, \ldots, f_{m}\right)\right\|_{L^{q(X, \mu)}} \leq C \prod_{j=1}^{m}\left\|f_{j}\right\|_{L^{p_{j}(X, \mu)}} ;
$$

(b) if $p_{j}=1$ for some $j$,

$$
\left\|\mathcal{I}_{\alpha, m}\left(f_{1}, \ldots, f_{m}\right)\right\|_{L^{q, \infty}(X, \mu)} \leq C \prod_{j=1}^{m}\left\|f_{j}\right\|_{L^{p_{j}}(X, \mu)} .
$$

Proof This lemma can follow the same argument that, for the classical setting, was given by Kenig and Stein [5]. We may assume that all $1 \leq p_{i}<\infty$. One can find $0<\alpha_{i}<1 / p_{i}$ such that $\alpha=\sum_{i=1}^{m} \alpha_{i}$. Set $1 / q_{i}=1 / p_{i}-\alpha_{i}$, since $1 / q=\sum_{i=1}^{m} 1 / q_{i}, 0<\alpha_{i} \leq 1,1<q_{i}<\infty$, and

$$
\rho\left(x, y_{1}\right)^{1-\alpha_{1}} \rho\left(x, y_{2}\right)^{1-\alpha_{2}} \cdots \rho\left(x, y_{m}\right)^{1-\alpha_{m}} \leq\left(\rho\left(x, y_{1}\right)+\cdots+\rho\left(x, y_{m}\right)\right)^{m-\alpha} .
$$

It follows that

$$
\mathcal{I}_{\alpha, m}\left(f_{1}, \ldots, f_{m}\right)(x) \leq \prod_{i=1}^{m} \mathcal{I}_{\alpha_{i}}\left(f_{i}\right)(x)
$$

Then, by the Hölder inequality and Theorem 2.1, we obtain the lemma. In fact, one could also get the lemma from [13] (or Remark 1.3, p.290 in [13]).

Now we give the proof of Theorem 1.5.

Proof of Theorem 1.5 Let $B=B\left(x_{0}, r\right)$ be the ball in $B \in \mathscr{B}(\mu)$, with center $x_{0} \in X$ and radius $r>0$, and let $B^{*}=B\left(x_{0}, 2 a_{1} r\right)$. For $f_{i} \in L^{p_{i}, \phi_{i}}(X, \mu)$, we split it as $f_{i}=f_{i}^{0}+f_{i}^{\infty}$, where $f_{i}^{0}=f_{i} \chi_{B^{*}}$ for $i=1, \ldots, m$. Using this decomposition, we get

$$
\left|\mathcal{I}_{\alpha, m}\left(f_{1}, \ldots, f_{m}\right)(x)\right| \leq\left|\mathcal{I}_{\alpha, m}\left(f_{1}^{0}, \ldots, f_{m}^{0}\right)(x)\right|+\sum^{\prime}\left|\mathcal{I}_{\alpha, m}\left(f_{1}^{\tau_{1}}, \ldots, f_{m}^{\tau_{m}}\right)(x)\right|
$$

where each term in $\sum^{\prime}$ contains at least one $\tau_{i}=\infty$. 
Then it suffices to show

$$
\frac{1}{\psi\left(\mu\left(k B^{*}\right)\right)}\left(\frac{1}{\mu\left(k B^{*}\right)} \int_{B}\left|\mathcal{I}_{\alpha, m}\left(f_{1}^{\tau_{1}}, \ldots, f_{m}^{\tau_{m}}\right)\right|^{q} d \mu\right)^{\frac{1}{q}} \leq C \prod_{i=1}^{m}\left\|f_{i}\right\|_{L^{p_{i}}, \phi_{i}(X, \mu)}
$$

for some $k>a_{1}$ and for each $\tau_{i} \in\{0, \infty\}$.

Let us first estimate for the case $\tau_{1}=\cdots=\tau_{m}=0$. From the definition of $L^{p_{i}, \phi_{i}}(X, \mu)$ we have

$$
\left(\int_{B^{*}}\left|f_{i}(x)\right|^{p_{i}} d \mu(x)\right)^{\frac{1}{p_{i}}} \leq C\left\|f_{i}\right\|_{L^{p_{i}, \phi_{i}(X, \mu)}} \phi_{i}\left(\mu\left(k B^{*}\right)\right) \mu\left(k B^{*}\right)^{\frac{1}{p_{i}}}
$$

From this and by the $L^{p_{1}}(X, \mu) \times \cdots \times L^{p_{m}}(X, \mu) \rightarrow L^{q}(X, \mu)$ boundedness of $\mathcal{I}_{\alpha, m}$, Lemma 2.2, we have

$$
\begin{aligned}
& \left(\frac{1}{\mu\left(k B^{*}\right)} \int_{B}\left|\mathcal{I}_{\alpha, m}\left(f_{1}^{0}, \ldots, f_{m}^{0}\right)(x)\right|^{q} d \mu(x)\right)^{\frac{1}{q}} \\
& \quad \leq \frac{C}{\mu\left(k B^{*}\right)^{\frac{1}{q}}} \prod_{i=1}^{m}\left\|f_{i}^{0}\right\|_{L^{p_{i}(X, \mu)}} \leq C \mu\left(k B^{*}\right)^{\alpha} \phi\left(\mu\left(k B^{*}\right)\right) \prod_{i=1}^{m}\left\|f_{i}\right\|_{L^{p_{i}, \phi_{i}(X, \mu)}} \\
& \quad \leq C \psi\left(\mu\left(k B^{*}\right)\right) \prod_{i=1}^{m}\left\|f_{i}\right\|_{L^{p_{i}, \phi_{i}(X, \mu)}},
\end{aligned}
$$

which implies that in the case all $\tau_{i}=0$, inequality (2.1) holds.

To estimate (2.1) for the case $\tau_{1}=\cdots=\tau_{m}=\infty$, let $x \in B$, then

$$
\left|\mathcal{I}_{\alpha, m}\left(f_{1}^{\infty}, \ldots, f_{m}^{\infty}\right)(x)\right| \leq \int_{\left(X \backslash B^{*}\right)^{m}} \frac{\left|f_{1}\left(y_{1}\right) \cdots f_{m}\left(y_{m}\right)\right| d \mu\left(y_{1}\right) \cdots d \mu\left(y_{m}\right)}{\left(\rho\left(x, y_{1}\right)+\cdots+\rho\left(x, y_{m}\right)\right)^{m-\alpha}}
$$

Note, for $x \in B$ and $y_{i} \in X \backslash B^{*}$, we get by (1.1) that

$$
2 a_{1} r<\rho\left(x_{0}, y_{i}\right) \leq a_{1}\left(\rho\left(x_{0}, x\right)+\rho\left(x, y_{i}\right)\right) \leq a_{1}\left(r+\rho\left(x, y_{i}\right)\right),
$$

hence $\rho\left(x, y_{i}\right) \geq r$. This and condition (1.2) of the measure $\mu$ imply that

$$
\rho\left(x, y_{i}\right) \geq r \geq\left(2 a_{1} C_{0} k\right)^{-1} \mu\left(k B^{*}\right):=r^{*}
$$

and so

$$
\left(X \backslash B^{*}\right)^{m} \subseteq \prod_{i=1}^{m}\left\{y_{i}: \rho\left(x, y_{i}\right) \geq r^{*}\right\} \subseteq\left\{\left(y_{1}, y_{2}, \ldots, y_{m}\right): \sum_{i=1}^{m} \rho\left(x, y_{i}\right) \geq r^{*}\right\}
$$

Hence we can derive that

$$
\begin{aligned}
& \left|\mathcal{I}_{\alpha, m}\left(f_{1}^{\infty}, \ldots, f_{m}^{\infty}\right)(x)\right| \\
& \quad \leq \int_{\sum_{i=1}^{m} \rho\left(x, y_{i}\right) \geq r^{*}} \frac{\left|f_{1}\left(y_{1}\right) \cdots f_{m}\left(y_{m}\right)\right| d \mu\left(y_{1}\right) \cdots d \mu\left(y_{m}\right)}{\left(\rho\left(x, y_{1}\right)+\cdots+\rho\left(x, y_{m}\right)\right)^{m-\alpha}}
\end{aligned}
$$




$$
\begin{aligned}
& =\sum_{j=0}^{\infty} \int_{2 r^{*} \leq \sum_{i=1}^{m} \rho\left(x, y_{i}\right)<2^{j+1} r^{*}} \frac{\left|f_{1}\left(y_{1}\right) \cdots f_{m}\left(y_{m}\right)\right| d \mu\left(y_{1}\right) \cdots d \mu\left(y_{m}\right)}{\left(\rho\left(x, y_{1}\right)+\cdots+\rho\left(x, y_{m}\right)\right)^{m-\alpha}} \\
& \leq \sum_{j=0}^{\infty} \frac{1}{\left(2 j r^{*}\right)^{m-\alpha}} \prod_{i=1}^{m} \int_{\rho\left(x, y_{i}\right)<2^{j+1} r^{*}}\left|f_{i}\left(y_{i}\right)\right| d \mu\left(y_{i}\right) .
\end{aligned}
$$

Using the Hölder inequality and the inequality similar to (2.2), we can see that the inequality above can be controlled by

$$
\begin{aligned}
C & \sum_{j=0}^{\infty} \frac{1}{\left(2 j r^{*}\right)^{m-\alpha}} \prod_{i=1}^{m}\left(\int_{B\left(x, j^{j+1} r^{*}\right)}\left|f_{i}\left(y_{i}\right)\right|^{p_{i}} d \mu\left(y_{i}\right)\right)^{\frac{1}{p_{i}}}\left(\mu\left(B\left(x, 2^{j+1} r^{*}\right)\right)\right)^{1-\frac{1}{p_{i}}} \\
& \leq C \sum_{j=0}^{\infty} \frac{1}{\left(2 j r^{*}\right)^{m-\alpha}} \prod_{i=1}^{m} \phi_{i}\left(\mu\left(B\left(x, k 2^{j+1} r^{*}\right)\right)\right) \mu\left(B\left(x, k 2^{j+1} r^{*}\right)\right)\left\|f_{i}\right\|_{L^{p_{i}}, \phi_{i}(X, \mu)} \\
& \leq C \sum_{j=0}^{\infty} \frac{1}{\left(2 r^{*}\right)^{m-\alpha}} \prod_{i=1}^{m} \phi_{i}\left(2^{j} \mu\left(k B^{*}\right)\right) 2^{j} \mu\left(k B^{*}\right)\left\|f_{i}\right\|_{L^{p_{i}}, \phi_{i}(X, \mu)} \\
& \leq C \prod_{i=1}^{m}\left[\sum_{j=0}^{\infty}\left(2^{j} \mu\left(k B^{*}\right)\right)^{\alpha / m} \phi_{i}\left(2^{j} \mu\left(k B^{*}\right)\right)\right]\left\|f_{i}\right\|_{L^{p_{i}}, \phi_{i}(X, \mu)},
\end{aligned}
$$

where, in the second inequality, we have utilized the non-decreasing of function $r^{\frac{1}{p_{i}}} \phi_{i}(r)$ and the fact $\mu\left(B\left(x, k 2^{j+1} r^{*}\right)\right) \leq C_{0} k 2^{j+1} r^{*} \leq 2^{j} \mu\left(k B^{*}\right)$. Recall conditions (1.4) (or (1.6)) and (1.5) for the function $\phi_{i}$, one sees that

$$
\begin{aligned}
\sum_{j=0}^{\infty}\left(2^{j} \mu\left(k B^{*}\right)\right)^{\alpha / m} \phi_{i}\left(2^{j} \mu\left(k B^{*}\right)\right) & \leq C \sum_{j=0}^{\infty} \int_{2^{j} \mu\left(k B^{*}\right)}^{j^{j+1} \mu\left(k B^{*}\right)} t^{\alpha / m-1} \phi_{i}(t) d t \\
& \leq C \int_{\mu\left(B^{*}\right)}^{\infty} t^{\alpha / m-1} \phi_{i}(t) d t \leq C \mu\left(k B^{*}\right)^{\alpha / m} \phi_{i}\left(\mu\left(k B^{*}\right)\right) .
\end{aligned}
$$

Hence we obtain the pointwise estimate

$$
\left|\mathcal{I}_{\alpha, m}\left(f_{1}^{\infty}, \ldots, f_{m}^{\infty}\right)(x)\right| \leq C \psi\left(\mu\left(k B^{*}\right)\right) \prod_{i=1}^{m}\left\|f_{i}\right\|_{L^{p_{i}}, \phi_{i}(X, \mu)}
$$

for $x \in B$, which follows from inequality (2.1) for the case all $\tau_{i}=\infty$.

It is left to consider the case $\tau_{i_{1}}=\cdots=\tau_{i_{l}}=0$ for some $\left\{i_{1}, \ldots, i_{l}\right\} \subset\{1, \ldots, m\}$ and $1 \leq$ $l<m$. For this case, we can write for $x \in B$ that

$$
\begin{aligned}
\left|\mathcal{I}_{\alpha, m}\left(f_{1}^{\tau_{1}}, \ldots, f_{m}^{\tau_{m}}\right)(x)\right| & \leq \int_{X^{m}} \frac{\left|f_{1}\left(y_{1}\right) \cdots f_{m}\left(y_{m}\right)\right| d \mu\left(y_{1}\right) \cdots d \mu\left(y_{m}\right)}{\left(\rho\left(x, y_{1}\right)+\cdots+\rho\left(x, y_{m}\right)\right)^{m-\alpha}} \\
& \leq \int_{\left(B^{*}\right)_{l}} \prod_{i \in\left\{i_{1}, \ldots, i_{l}\right\}}\left|f_{i}\left(y_{i}\right)\right| d \mu\left(y_{i}\right) \cdot \int_{\left(X \backslash B^{*}\right)^{m-l}} \frac{\prod_{i \notin\left\{i_{1}, \ldots, i_{l}\right\}}\left|f_{i}\left(y_{i}\right)\right| d \mu\left(y_{i}\right)}{\left(\sum_{i \notin\left\{i_{1}, \ldots, i_{l}\right\}} \rho\left(x, y_{i}\right)\right)^{m-\alpha}} \\
& :=A_{1}(x) \cdot A_{2}(x) .
\end{aligned}
$$


To estimate $A_{1}(x)$, we use the Hölder inequality to give that, for any $x \in B$,

$$
\begin{aligned}
A_{1}(x) & =\prod_{i \in\left\{i_{1}, \ldots, i_{l}\right\}} \int_{B^{*}}\left|f_{i}\left(y_{i}\right)\right| d \mu\left(y_{i}\right) \\
& \leq C \prod_{i \in\left\{i_{1}, \ldots, i_{l}\right\}}\left(\int_{B^{*}}\left|f_{i}\right|^{p_{i}} d \mu\left(y_{i}\right)\right)^{\frac{1}{p_{i}}} \mu\left(B^{*}\right)^{1-\frac{1}{p_{i}}} \\
& \leq C \mu\left(k B^{*}\right)^{l} \prod_{i \in\left\{i_{1}, \ldots, i_{l}\right\}}\left(\phi_{i}\left(\mu\left(k B^{*}\right)\right)\left\|f_{i}\right\|_{L^{p_{i}, \phi_{i}}(X, \mu)}\right) .
\end{aligned}
$$

Estimating $A_{2}(x)$, by the same idea used for the case all $\tau_{i}=\infty$ above, we get for any $x \in B$ that

$$
\begin{aligned}
A_{2}(x) & \leq \sum_{j=0}^{\infty} \frac{1}{\left(2 j r^{*}\right)^{m-\alpha}} \prod_{i \notin\left\{i_{1}, \ldots, i_{l}\right\}} \int_{B\left(x, 2^{j+1} r^{*}\right)}\left|f_{i}\left(y_{i}\right)\right| d \mu\left(y_{i}\right) \\
& \leq C \sum_{j=0}^{\infty} \frac{1}{\left(2 r^{*}\right)^{m-\alpha}} \prod_{i \notin\left\{i_{1}, \ldots, i_{l}\right\}} \phi_{i}\left(2^{j} \mu\left(k B^{*}\right)\right) 2^{j} \mu\left(k B^{*}\right)\left\|f_{i}\right\|_{L^{p_{i}, \phi_{i}(X, \mu)}} \\
& \leq C \sum_{j=0}^{\infty} \frac{1}{\left(2^{j} \mu\left(k B^{*}\right)\right)^{l-\alpha l / m}} \prod_{i \notin\left\{i_{1}, \ldots, i_{l}\right\}} \phi_{i}\left(2^{j} \mu\left(k B^{*}\right)\right)\left(2^{j} \mu\left(k B^{*}\right)\right)^{\alpha / m}\left\|f_{i}\right\|_{L^{p_{i}, \phi_{i}(X, \mu)}} .
\end{aligned}
$$

Noting $l-l \alpha / m>0$ and using condition (1.5), we have

$$
\begin{aligned}
A_{2}(x) & \leq C\left(\mu\left(k B^{*}\right)\right)^{\alpha l / m-l} \prod_{i \notin\left\{i_{1}, \ldots, i_{l}\right\}} \phi_{i}\left(\mu\left(k B^{*}\right)\right) \mu\left(k B^{*}\right)^{\alpha / m}\left\|f_{i}\right\|_{L^{p_{i}, \phi_{i}(X, \mu)}} \\
& \leq C\left(\mu\left(k B^{*}\right)\right)^{\alpha-l} \prod_{i \notin\left\{i_{1}, \ldots, i_{l}\right\}} \phi_{i}\left(\mu\left(k B^{*}\right)\right)\left\|f_{i}\right\|_{L^{p_{i}}, \phi_{i}(X, \mu)} .
\end{aligned}
$$

Therefore, for $x \in B$, we have

$$
\begin{aligned}
\left|\mathcal{I}_{\alpha, m}\left(f_{1}^{\tau_{1}}, \ldots, f_{m}^{\tau_{m}}\right)(x)\right| & \leq A_{1}(x) \cdot A_{2}(x) \\
& \leq C \mu\left(k B^{*}\right)^{\alpha} \prod_{i=1}^{m} \phi_{i}\left(\mu\left(k B^{*}\right)\right)\left\|f_{i}\right\|_{L^{p_{i}, \phi_{i}}(X, \mu)} \\
& \leq C \psi\left(\mu\left(k B^{*}\right)\right) \prod_{i=1}^{m}\left\|f_{i}\right\|_{L^{p_{i}, \phi_{i}}(X, \mu)} .
\end{aligned}
$$

Hence we obtain the desired inequality (2.1) for any cases. The proof of the theorem is complete.

\section{Proof of Theorems 1.7 and 1.9}

In this section we first investigate the boundedness of the $m$-linear Calderón-Zygmund operator $\mathcal{T}$ on the product of spaces $L^{p_{i}, \phi_{i}}(X, \mu)$ for $i=1,2, \ldots, m$.

Proof of Theorem 1.7 We also let $B=B\left(x_{0}, r\right)$ be the ball in $\mathscr{B}(\mu)$, with center $x_{0} \in X$ and radius $r>0$, and let $B^{*}=B\left(x_{0}, 2 a_{1} r\right)$. For the admissible $f_{i} \in L^{p_{i}, \phi_{i}}(X, \mu)$, without loss of 
generality, we may initially assume that $f_{i}$ are all smooth boundedly supported functions, which are dense in $L^{p_{i}}(X, \mu)$, and let $f_{i} \in L^{p_{i}}(X, \mu) \cap L^{p_{i}, \phi_{i}}(X, \mu)$, then $\mathcal{T}\left(f_{1}, \ldots, f_{m}\right)$ is a welldefined function belonging to $L^{p}(X, \mu)$. If we split each $f_{i}$ as $f_{i}=f_{i}^{0}+f_{i}^{\infty}$, where $f_{i}^{0}=f_{i} \chi_{B^{*}}$ for $i=1, \ldots, m$, and utilize the multi-linearity of $\mathcal{T}$, we have the following decomposition,

$$
\left|\mathcal{T}\left(f_{1}, \ldots, f_{m}\right)(x)\right| \leq\left|\mathcal{T}\left(f_{1}^{0}, \ldots, f_{m}^{0}\right)(x)\right|+\sum^{\prime}\left|\mathcal{T}\left(f_{1}^{\tau_{1}}, \ldots, f_{m}^{\tau_{m}}\right)(x)\right|,
$$

where each term in $\sum^{\prime}$ contains at least one $\tau_{i}=\infty$.

Noting that $\mathcal{T}$ is bounded from $L^{p_{1}}(X, \mu) \times \cdots \times L^{p_{m}}(X, \mu) \rightarrow L^{p}(X, \mu)$, we have

$$
\begin{aligned}
& \left(\frac{1}{\mu\left(k B^{*}\right)} \int_{B}\left|\mathcal{T}\left(f_{1}^{0}, \ldots, f_{m}^{0}\right)(x)\right|^{p} d \mu(x)\right)^{\frac{1}{p}} \\
& \quad \leq \frac{C}{\mu\left(k B^{*}\right)^{\frac{1}{p}}} \prod_{i=1}^{m}\left\|f_{i}^{0}\right\|_{L^{p_{i}}(X, \mu)} \\
& \quad \leq C \phi\left(\mu\left(k B^{*}\right)\right) \prod_{i=1}^{m}\left\|f_{i}\right\|_{L^{p_{i}, \phi_{i}(X, \mu)}} .
\end{aligned}
$$

For the case $\tau_{1}=\cdots=\tau_{m}=\infty$, we note that for $x \in B$ and $y_{i} \in X \backslash B^{*}$, one can deduce from the properties of the quasi-metric $\rho$ that

$$
\frac{1}{2 a_{1}} \rho\left(x_{0}, y_{i}\right) \leq \rho\left(x, y_{i}\right) \leq\left(\frac{a_{0}}{2}+a_{1}\right) \rho\left(x_{0}, y_{i}\right)
$$

Thus we can observe that

$$
\frac{1}{\left(\sum_{i=1}^{m} \rho\left(x, y_{i}\right)\right)^{m}} \simeq \frac{1}{\left(\sum_{i=1}^{m} \rho\left(x_{0}, y_{i}\right)\right)^{m}}=m \int_{\rho\left(x_{0}, y_{1}\right)+\cdots+\rho\left(x_{0}, y_{m}\right)}^{\infty} \frac{d l}{l^{m+1}} .
$$

This, together with the Fubini theorem, we have, for $x \in B$,

$$
\begin{aligned}
\left|\mathcal{T}\left(f_{1}^{\infty}, \ldots, f_{m}^{\infty}\right)(x)\right| & \leq \int_{\left(X \backslash B^{*}\right)^{m}} \frac{\prod_{i=1}^{m}\left|f_{i}\left(y_{i}\right)\right| d \mu\left(y_{i}\right)}{\left(\sum_{i=1}^{m} \rho\left(x, y_{i}\right)\right)^{m}} \\
& \leq C \int_{\sum_{i=1}^{m} \rho\left(x_{0}, y_{i}\right) \geq 2 a_{1} r}\left(\int_{\rho\left(x_{0}, y_{1}\right)+\cdots+\rho\left(x_{0}, y_{m}\right)}^{\infty} \frac{d l}{l^{m+1}}\right) \prod_{i=1}^{m}\left|f_{i}\left(y_{i}\right)\right| d \mu\left(y_{i}\right) \\
& \leq C \int_{2 a_{1} r}^{\infty} \frac{1}{l^{m+1}}\left(\int_{\sum_{i=1}^{m} \rho\left(x_{0}, y_{i}\right)<l} \prod_{i=1}^{m}\left|f_{i}\left(y_{i}\right)\right| d \mu\left(y_{i}\right)\right) d l \\
& \leq C \int_{2 a_{1} r}^{\infty} \frac{1}{l^{m+1}}\left(\prod_{i=1}^{m} \int_{\rho\left(x_{0}, y_{i}\right)<l}\left|f_{i}\left(y_{i}\right)\right| d \mu\left(y_{i}\right)\right) d l .
\end{aligned}
$$

Noting $\mu\left(k B^{*}\right) \leq C_{0} 2 k a_{1} r$ and applying the Hölder inequality, we see that the inequality above is bounded by

$$
\begin{aligned}
& C \int_{\mu\left(k B^{*}\right) / C_{0} k}^{\infty} \frac{1}{l^{m+1}} \prod_{i=1}^{m}\left(\int_{B\left(x_{0}, l\right)}\left|f_{i}\left(y_{i}\right)\right|^{p_{i}} d \mu\left(y_{i}\right)\right)^{\frac{1}{p_{i}}} \mu\left(B\left(x_{0}, l\right)\right)^{1-\frac{1}{p_{i}}} d l \\
& \quad \leq C \int_{\mu\left(k B^{*}\right) / C_{0} k}^{\infty} \frac{1}{l^{m+1}} \prod_{i=1}^{m}\left(\left\|f_{i}\right\|_{L^{p_{i}}, \phi_{i}(X, \mu)} \phi_{i}\left(\mu\left(k B\left(x_{0}, l\right)\right)\right) \mu\left(k B\left(x_{0}, l\right)\right)\right) d l
\end{aligned}
$$


which, by using the non-decreasing of function $r \phi_{i}(r)$, is controlled by

$$
\begin{aligned}
& C \int_{\mu\left(k B^{*}\right)}^{\infty}\left(\prod_{i=1}^{m} \phi_{i}(l)\right) \frac{d l}{l} \cdot \prod_{i=1}^{m}\left\|f_{i}\right\|_{L^{p_{i}, \phi_{i}(X, \mu)}} \\
& \quad \leq C\left(\prod_{i=1}^{m} \int_{\mu\left(k B^{*}\right)}^{\infty} \phi_{i}(l)^{\frac{p_{i}}{p}} \frac{d l}{l}\right)^{\frac{p}{p_{i}}} \cdot \prod_{i=1}^{m}\left\|f_{i}\right\|_{L^{p_{i}, \phi_{i}(X, \mu)}} \\
& \quad \leq C \phi\left(\mu\left(k B^{*}\right)\right) \prod_{i=1}^{m}\left\|f_{i}\right\|_{L^{p_{i}, \phi_{i}(X, \mu)}} .
\end{aligned}
$$

Therefore, we get for $x \in B$ that

$$
\left|\mathcal{T}\left(f_{1}^{\infty}, \ldots, f_{m}^{\infty}\right)(x)\right| \leq C \phi\left(\mu\left(k B^{*}\right)\right) \prod_{i=1}^{m}\left\|f_{i}\right\|_{L^{p_{i}, \phi_{i}(X, \mu)}}
$$

It is left to consider the case that there is $1 \leq l<m$ and $\left\{i_{1}, \ldots, i_{l}\right\} \subset\{1, \ldots, m\}$ such that $\tau_{i}=0$ if $i \in\left\{i_{1}, \ldots, i_{l}\right\}$, and $\tau_{i}=\infty$ if $i \notin\left\{i_{1}, \ldots, i_{l}\right\}$. For $x \in B$, we can write that

$$
\begin{aligned}
& \left|\mathcal{T}\left(f_{1}^{\tau_{1}}, \ldots, f_{m}^{\tau_{m}}\right)(x)\right| \\
& \quad \leq \int_{\left(B^{*}\right)^{l}} \prod_{i \in\left\{i_{1}, \ldots, i_{l}\right\}}\left|f_{i}\left(y_{i}\right)\right| d \mu\left(y_{i}\right) \int_{\left(X \backslash B^{*}\right)^{m-l}} \frac{\prod_{i \notin\left\{i_{1}, \ldots, i_{l}\right\}}\left|f_{i}\left(y_{i}\right)\right| d \mu\left(y_{i}\right)}{\left(\sum_{i \notin\left\{i_{1}, \ldots, i_{l}\right\}} \rho\left(x, y_{i}\right)\right)^{m}} \\
& \quad:=E_{1}(x) \cdot E_{2}(x) .
\end{aligned}
$$

With the same argument as $A_{1}(x)$ we have

$$
E_{1}(x) \leq C \mu\left(k B^{*}\right)^{l} \prod_{i \in\left\{i_{1}, \ldots, i_{l}\right\}} \phi_{i}\left(\mu\left(k B^{*}\right)\right)\left\|f_{i}\right\|_{L^{p_{i}, \phi_{i}}(X, \mu)} .
$$

Using a similar argument as that for the estimate of $\mathcal{T}\left(f_{1}^{\infty}, \ldots, f_{m}^{\infty}\right)(x)$, we can deduce that

$$
\begin{aligned}
E_{2}(x) & \leq C \int_{\mu\left(k B^{*}\right)}^{\infty}\left(\prod_{i \notin\left\{i_{1}, \ldots, i_{l}\right\}} \phi_{i}(t)\right) \frac{d t}{t^{l+1}} \cdot \prod_{i \notin\left\{i_{1}, \ldots, i_{l}\right\}}\left\|f_{i}\right\|_{L^{p_{i}, \phi_{i}(X, \mu)}} \\
& \leq C \mu\left(k B^{*}\right)^{-l}\left[\prod_{i \notin\left\{i_{1}, \ldots, i_{l}\right\}}\left(\int_{\mu\left(k B^{*}\right)}^{\infty} \phi_{i}(t)^{\frac{p_{i}}{p}} \frac{d t}{t}\right)^{\frac{p}{p_{i}}}\right] \prod_{i \notin\left\{i_{1}, \ldots, i_{l}\right\}}\left\|f_{i}\right\|_{L^{p_{i}, \phi_{i}(X, \mu)}} \\
& \leq C \mu\left(k B^{*}\right)^{-l}\left[\prod_{i \notin\left\{i_{1}, \ldots, i_{l}\right\}} \phi_{i}\left(\mu\left(k B^{*}\right)\right)\right] \prod_{i \notin\left\{i_{1}, \ldots, i_{l}\right\}}\left\|f_{i}\right\|_{L^{p_{i}, \phi_{i}}(X, \mu)} .
\end{aligned}
$$

Hence we obtain that

$$
\left|\mathcal{T}\left(f_{1}^{\tau_{1}}, \ldots, f_{m}^{\tau_{m}}\right)(x)\right| \leq E_{1}(x) \cdot E_{2}(x) \leq C \phi\left(\mu\left(k B^{*}\right)\right) \prod_{i=1}^{m}\left\|f_{i}\right\|_{L^{p_{i}, \phi_{i}}(X, \mu)} .
$$


Therefore, combining inequalities (3.1), (3.2) and (3.3), we have

$$
\left(\frac{1}{\mu\left(k B^{*}\right)} \int_{B}\left|\mathcal{T}\left(f_{1}, \ldots, f_{m}\right)(x)\right|^{p} d \mu(x)\right)^{\frac{1}{p}} \leq C \phi\left(\mu\left(k B^{*}\right)\right) \prod_{i=1}^{m}\left\|f_{i}\right\|_{L^{p_{i}, \phi_{i}}(X, \mu)},
$$

which completes the proof of Theorem 1.7.

Remark 3.1 We have actually proved Theorem 1.7 in the case $f_{i} \in L^{p_{i}}(X, \mu) \cap L^{p_{i}, \phi_{i}}(X, \mu)$. Here we need give some remarks about the definition and boundedness of $\mathcal{T}\left(f_{1}, \ldots, f_{m}\right)$ with $f_{i} \in L^{p_{i}, \phi_{i}}(X, \mu)$ for $i=1,2, \ldots, m$. Fix any $x_{0} \in X$ and $R>0$, and use the same notations $f_{i}^{\tau_{i}}=f_{i} \chi_{B\left(x_{0}, 2 a_{1} R\right)}$ if $\tau_{i}=0$, and $f_{i}^{\tau_{i}}=f_{i}-f_{i}^{0}$ if $\tau_{i}=\infty$. Using a similar argument as (3.2) and (3.3), we have, if some $\tau_{i}=\infty$,

$$
\begin{aligned}
& \int_{X^{m}}\left|\mathcal{K}\left(x, y_{1}, \ldots, y_{m}\right) f_{1}^{\tau_{1}}\left(y_{1}\right) \cdots f_{m}^{\tau_{m}}\left(y_{m}\right)\right| d \mu\left(y_{1}\right) \cdots d \mu\left(y_{m}\right) \\
& \quad \leq C \phi\left(\mu\left(B\left(x_{0}, 3 a_{1}^{2} R\right)\right)\right) \prod_{i=1}^{m}\left\|f_{i}\right\|_{L^{p_{i}}, \phi_{i}(X, \mu)}
\end{aligned}
$$

with the constant $C$ independent of $R$, for all $f_{i} \in L^{p_{i}, \phi_{i}}(X, \mu), i=1, \ldots, m$, and $x \in B\left(x_{0}\right.$, $R) \subset X$.

In view of this fact, and if $\lim _{R \rightarrow \infty} \phi\left(\mu\left(B\left(x_{0}, 3 a_{1}^{2} R\right)\right)\right)=0$, then we can extend the definition of $\mathcal{T}$ for $f_{i} \in L^{p_{i}, \phi_{i}}(X, \mu)$ by

$$
\begin{aligned}
\mathcal{T} & \left(\tau_{1}^{\tau_{1}}, \ldots, f_{m}^{\tau_{m}}\right)(x) \\
= & \lim _{R \rightarrow \infty}\left(\mathcal{T}\left(f_{1}^{0}, \ldots, f_{m}^{0}\right)(x)\right. \\
& \left.+\sum_{\text {some } \tau_{i}=\infty} \int_{X^{m}}\left|\mathcal{K}\left(x, y_{1}, \ldots, y_{m}\right) f_{1}^{\tau_{1}}\left(y_{1}\right) \cdots f_{m}^{\tau_{m}}\left(y_{m}\right)\right| d \mu\left(y_{1}\right) \cdots d \mu\left(y_{m}\right)\right) .
\end{aligned}
$$

By the definition, it is easy to see that the following properties hold.

(1) If $\rho\left(x_{0}, x\right) \leq R_{0}$, then the terms in the brackets on the right-hand side of the equation above do not depend on $R$ as long as $R>R_{0}$.

(2) Suppose that $1<p_{1}, \ldots, p_{m}<\infty$, and if $f_{i} \in L^{p_{i}}(X, \mu) \cap L^{p_{i}, \phi_{i}}(X, \mu)$, then the definitions of $\mathcal{T}\left(f_{1}, \ldots, f_{m}\right)$ for $f_{i} \in L^{p_{i}}(X, \mu)$ and for $f_{i} \in L^{p_{i}, \phi_{i}}(X, \mu)$ coincide.

(3) Theorem 1.7 holds for any admissible $f_{i} \in L^{p_{i}, \phi_{i}}(X, \mu), i=1, \ldots, m$.

Finally, we consider the multi-sublinear maximal function $\mathcal{M}_{\kappa}\left(f_{1}, \ldots, f_{m}\right)(x)$, which is strictly smaller than the $m$-fold produce of the maximal function $M_{\kappa}\left(f_{i}\right)(x)$. Hence we have the following lemma.

Lemma 3.2 If $\kappa>a_{1}^{2}$ and $p, p_{i}>1$, and $\frac{1}{p}=\frac{1}{p_{1}}+\cdots+\frac{1}{p_{m}}$, then there exists a constant $C$ independent of $f_{i}$ such that

$$
\left\|\mathcal{M}_{\kappa}\left(f_{1}, \ldots, f_{m}\right)\right\|_{L^{p}(X, \mu)} \leq C \prod_{i=1}^{m}\left\|f_{i}\right\|_{L^{p_{i}(X, \mu)}} .
$$


Proof of Theorem 1.9 With the same notions, we decompose each $f_{i} \in L^{p_{i}, \phi_{i}}(X, \mu)$ according to the ball $B^{*}:=B\left(x_{0}, a_{1}(1+\lambda) r\right)$ as $f_{i}=f_{i}^{0}+f_{i}^{\infty}$, where $\lambda$ is a large positive constant that will be determined later. We have

$$
\left|\mathcal{M}_{\kappa}\left(f_{1}, \ldots, f_{m}\right)(x)\right| \leq\left|\mathcal{M}_{\kappa}\left(f_{1}^{0}, \ldots, f_{m}^{0}\right)(x)\right|+\sum^{\prime}\left|\mathcal{M}_{\kappa}\left(f_{1}^{\tau_{1}}, \ldots, f_{m}^{\tau_{m}}\right)(x)\right|
$$

where each term in $\sum^{\prime}$ contains at least one $\tau_{i} \neq 0$.

It follows from Lemma 3.2 that for any $k>a_{1}$ and $\kappa>a_{1}^{2}$,

$$
\begin{aligned}
& \left(\frac{1}{\mu\left(k B^{*}\right)} \int_{B\left(x_{0}, r\right)}\left|\mathcal{M}_{\kappa}\left(f_{1}^{0}, \ldots, f_{m}^{0}\right)(x)\right|^{p} d \mu(x)\right)^{\frac{1}{p}} \\
& \quad \leq C \phi\left(\mu\left(k B^{*}\right)\right) \prod_{i=1}^{m}\left\|f_{i}\right\|_{L^{p_{i}, \phi_{i}(X, \mu)}} .
\end{aligned}
$$

It is left to study the case $\tau_{i_{1}}=\cdots=\tau_{i_{l}}=0$ and $\tau_{i_{l+1}}=\cdots=\tau_{i_{m}}=\infty$ for some $1 \leq l<m$. Hence for $x \in B\left(x_{0}, r\right)$ we have

$$
\begin{aligned}
\mathcal{M}_{\kappa}\left(f_{1}^{\tau_{1}}, \ldots, f_{m}^{\tau_{m}}\right)(x)= & \sup _{x \in D \in \mathscr{B}(\mu)} \prod_{i=1}^{m} \frac{1}{\mu(\kappa D)} \int_{D}\left|f_{i}^{\tau_{i}}\left(y_{i}\right)\right| d \mu\left(y_{i}\right) \\
\leq & \sup _{x \in D \in \mathscr{B}(\mu)} \prod_{i \in\left\{i_{1}, \ldots, i_{l}\right\}} \frac{1}{\mu(\kappa D)} \int_{D}\left|f_{i}^{0}\left(y_{i}\right)\right| d \mu\left(y_{i}\right) \\
& \cdot \prod_{i \notin\left\{i_{1}, \ldots, i_{l}\right\}} \frac{1}{\mu(\kappa D)} \int_{D}\left|f_{i}^{\infty}\left(y_{i}\right)\right| d \mu\left(y_{i}\right) .
\end{aligned}
$$

Let $r_{D}$ be the radius of the ball $D$ and $c_{D}$ be the center of $D$. We note that on the right-hand side of the inequality above, the balls $D$ in the integrals must satisfy that $x \in D \cap B\left(x_{0}, r\right)$ and some $y_{i_{m}} \in D \cap\left(X \backslash B^{*}\right)$, which implies

$$
\begin{aligned}
& a_{1} \rho\left(x, y_{i_{m}}\right) \geq \rho\left(x_{0}, y_{i_{m}}\right)-a_{1} \rho\left(x_{0}, x\right) \geq a_{1} \lambda r, \\
& \rho\left(x, y_{i_{m}}\right) \leq a_{1} a_{0} \rho\left(c_{D}, x\right)+a_{1} \rho\left(c_{D}, y_{i_{m}}\right) \leq a_{1}\left(1+a_{0}\right) r_{D} .
\end{aligned}
$$

Further, a simple calculus yields

$$
B:=B\left(x_{0}, r\right) \subset\left(a_{1}+a_{1}^{3}\left(1+a_{0}\right)^{2} \lambda^{-1}\right) D \subset \frac{\kappa+a_{1}^{2}}{2 a_{1}} D
$$

as long as we take $\lambda$ big enough, because of $\kappa>a_{1}^{2}$. Thus,

$$
\mathcal{M}_{\kappa}\left(f_{1}^{\tau_{1}}, \ldots, f_{m}^{\tau_{m}}\right)(x) \leq \sup _{B \subset D \in \mathscr{B}(\mu)} \prod_{i=1}^{m} \frac{1}{\mu\left(\frac{2 a_{1} \kappa}{\kappa+a_{1}^{2}} D\right)} \int_{D}\left|f_{i}^{\tau_{i}}\left(y_{i}\right)\right| d \mu\left(y_{i}\right)
$$


If let $k=2 a_{1} \kappa /\left(\kappa+a_{1}^{2}\right)$, then $k>a_{1}$, and we can get from the Hölder inequality and condition (1.6) on $\phi_{i}$ that

$$
\begin{aligned}
\mathcal{M}_{\kappa}\left(f_{1}^{\tau_{1}}, \ldots, f_{m}^{\tau_{m}}\right)(x) & \leq \sup _{B \subset D \in \mathscr{B}(\mu)} \prod_{i=1}^{m} \frac{1}{\mu(k D)} \int_{D}\left|f_{i}^{\tau_{i}}\left(y_{i}\right)\right| d \mu\left(y_{i}\right) \\
& \leq C \sup _{B \subset D \in \mathscr{B}(\mu)} \prod_{i=1}^{m}\left\|f_{i}\right\|_{L^{p_{i}}, \phi_{i}(X, \mu)} \phi_{i}(\mu(k D)) \\
& \leq C \phi(\mu(k B)) \prod_{i=1}^{m}\left\|f_{i}\right\|_{L^{p_{i}}, \phi_{i}(X, \mu)} .
\end{aligned}
$$

The theorem is proved.

\section{Competing interests}

The authors declare that they have no competing interests.

\section{Authors' contributions}

XT finished the proof and the revised writing work. SH gave XT the first version of the manuscript. All authors read and approved the final manuscript.

\section{Author details}

${ }^{1}$ Department of Mathematics, Zhejiang University of Science and Technology, Hangzhou, Zhejiang 310023, P.R. China.

${ }^{2}$ Department of Mathematics, Hangzhou Normal University, Hangzhou, Zhejiang 310023, P.R. China.

\section{Acknowledgements}

The authors thank the anonymous referee for reading the paper carefully and giving several useful suggestions. The research was partially supported by the National Nature Science Foundation of China under grants \#11171306 and $\# 11071065$, and it was partially sponsored by the Scientific Project of Zhejiang Provincial Science Technology Department under grant \#2011C33012 and the Nature Science Foundation of Zhejiang Province under grant \#LY12A01024.

\section{Received: 5 May 2012 Accepted: 5 July 2013 Published: 22 July 2013}

\section{References}

1. Adams, DR: A note on Riesz potentials. Duke Math. J. 42(4), 765-778 (1975)

2. Chiarenza, F, Frasca, M: Morrey spaces and Hardy-Littlewood maximal function. Rend. Semin. Mat. Univ. Padova 7(3-4), 273-279 (1987)

3. Sawano, Y, Tanaka, H: Morrey spaces for non-doubling measures. Acta Math. Sin. Engl. Ser. 21(6), 1535-1544 (2005)

4. Sawano, Y: Generalized Morrey spaces for non-doubling measures. Nonlinear Differ. Equ. Appl. 15(4), 413-425 (2008)

5. Kenig, CE, Stein, EM: Multilinear estimates and fractional integration. Math. Res. Lett. 6(1), 1-15 (1999)

6. Grafakos, L, Torres, H: Multilinear Calderón-Zygmund theory. Adv. Math. 165(1), 124-164 (2002)

7. Tao, X-X, Shi, Y-L, Zheng, T-T: Multilinear Riesz potential on Morrey-Herz spaces with non-doubling measures. J. Inequal. Appl. 2010, Article ID 731016 (2010)

8. Tao, X-X, Shi, Y-L, Zhang, S-Y: Boundedness of multilinear Riesz potential operators on product of Morrey spaces and Herz-Morrey spaces. Acta Math. Sin. 52(3), 535-548 (2009)

9. Tao, X-X, Shi, Y-L: Boundedness for multilinear fractional integral operators on Herz type spaces. Appl. Math. J. Chin. Univ. Ser. B 23(4), 437-446 (2008)

10. Tao, X-X, Shi, Y-L: Multilinear Riesz potential operators on Herz-type spaces and generalized Morrey spaces. Hokkaido Math. J. 38(3), 635-662 (2009)

11. Shi, Y-L, Tao, X-X: Some multilinear operators on generalized Morrey spaces with non-doubling measures. J. Korean Math. Soc. 49(4), 907-925 (2012)

12. Tao, X-X, Zhang, H-H: On the boundedness of multilinear operators on weighted Herz-Morrey spaces. Taiwan. J. Math. $15(4), 1527-1543(2011)$

13. Tao, X-X, Zheng, T-T: Multilinear commutators of fractional integrals over Morrey spaces with non-doubling measures Nonlinear Differ. Equ. Appl. 18(3), 287-308 (2011)

14. Tao, X-X, Shi, Y-L: Multilinear commutators of Calderón-Zygmund operator on $\lambda$-central Morrey spaces. Adv. Math (China) 40(1), 47-59 (2011)

15. lida, T, Sato, E, Sawano, Y, Tanaka, H: Multilinear fractional integrals on Morrey spaces. Acta Math. Sin. Engl. Ser. 28(7), 1375-1384 (2012)

16. Xu, J-S: Boundedness of multilinear singular integrals for non-doubling measures. J. Math. Anal. Appl. 327(1), 471-480 (2007)

17. Hytönen, T: A framework for non-homogeneous analysis on metric spaces, and the RBMO space of Tosa. Publ. Mat. 54(2), 485-504 (2010) 
18. Nazarov, F, Treil, S, Volberg, S: The Tb-theorem on non-homogeneous spaces that proves a conjecture of Vitushkin. Preprint No. 519, Center de Recerca Matemàtica, Barcelona (2002)

19. Tolsa, X: Painlevé's problem and the semiadditivity of analytic capacity. Acta Math. 190(1), 105-149 (2003)

20. Verdera, J: The fall of the doubling condition in Calderón-Zygmund theory. Publ. Mat. 46(Extra), $275-292$ (2002)

21. Edmunds, DE, Kokilashvili, V, Meskhi, A: Bounded and Compact Integral Operators. Kluwer Academic, Dordrecht (2002)

doi:10.1186/1029-242X-2013-330

Cite this article as: Tao and He: The boundedness of multilinear operators on generalized Morrey spaces over the quasi-metric space of non-homogeneous type. Journal of Inequalities and Applications 2013 2013:330.

Submit your manuscript to a SpringerOpen ${ }^{\circ}$ journal and benefit from:

- Convenient online submission

Rigorous peer review

- Immediate publication on acceptance

- Open access: articles freely available online

- High visibility within the field

- Retaining the copyright to your article

Submit your next manuscript at $>$ springeropen.com 